\begin{tabular}{|c|l|}
\hline Title & Rectification of elastic waves in a thin plate \\
\hline Author(s) & Tanaka, Y ukihiro; Murai, Takahiro; Nishiguchi, Norihiko \\
\hline Citation & $\begin{array}{l}\text { Journal of A pplied Physics, 111/2), 024507 } \\
\text { https://doi.org/10.1063/1.3677997 }\end{array}$ \\
\hline Issue Date & 2012-01-15 \\
\hline Doc URL & http://hdl.handle.net/2115/48515 \\
\hline Rights & $\begin{array}{l}\text { Copyright 2012 A merican Institute of Physics. This article may be downloaded for personal use only. A ny other use } \\
\text { requires prior permission of the author and the A merican Institute of Physics. The following article appeared in J. A ppl. } \\
\text { Phys. 111, 024507 (2012) and may be found at https://dx.doi.org/10.1063/1.3677997 }\end{array}$ \\
\hline Type & article \\
\hline File Information & JAP111-2_024507.pdf \\
\hline
\end{tabular}

Instructions for use 
AIP Applied Physics

\section{Rectification of elastic waves in a thin plate}

Yukihiro Tanaka, Takahiro Murai, and Norihiko Nishiguchi

Citation: J. Appl. Phys. 111, 024507 (2012); doi: 10.1063/1.3677997

View online: http://dx.doi.org/10.1063/1.3677997

View Table of Contents: http://jap.aip.org/resource/1/JAPIAU/v111/i2

Published by the American Institute of Physics.

\section{Related Articles}

Quantitative measurement of in-plane cantilever torsion for calibrating lateral piezoresponse force microscopy Rev. Sci. Instrum. 82, 113706 (2011)

Decay rate estimates for the quasi-linear Timoshenko system with nonlinear control and damping terms J. Math. Phys. 52, 093502 (2011)

Surface effects on frequency analysis of nanotubes using nonlocal Timoshenko beam theory

J. Appl. Phys. 108, 093503 (2010)

Effect of local solid reaction on diffusion-induced stress

J. Appl. Phys. 107, 103516 (2010)

The role of elastic flap deformation on fluid mixing in a microchannel

Phys. Fluids 22, 052003 (2010)

\section{Additional information on J. Appl. Phys.}

Journal Homepage: http://jap.aip.org/

Journal Information: http://jap.aip.org/about/about_the_journal

Top downloads: http://jap.aip.org/features/most_downloaded

Information for Authors: http://jap.aip.org/authors

\section{ADVERTISEMENT}

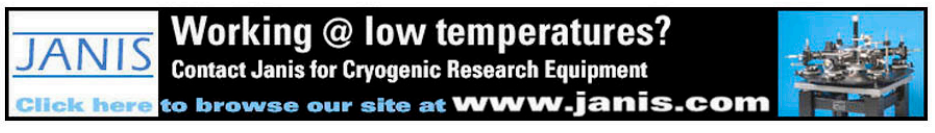




\title{
Rectification of elastic waves in a thin plate
}

\author{
Yukihiro Tanaka, ${ }^{\text {a) }}$ Takahiro Murai, and Norihiko Nishiguchi \\ Department of Applied Physics, Hokkaido University, Sapporo 060-8628, Japan
}

(Received 23 June 2011; accepted 16 December 2011; published online 25 January 2012)

\begin{abstract}
We propose a rectifier of elastic waves in a thin plate, which is made of an elastically isotropic material containing a periodic array of triangular holes as scatterers, and demonstrate numerically that it works both for the symmetric and anti-symmetric Lamb waves as well as shear horizontal waves. The rectification is caused by the geometric effects on wave scattering due to the asymmetric scatterers, while the interplay between the mode conversion and interference effects among the scattered waves owing to the periodic arrangement of scatterers complicates it. The mechanism makes it possible to rectify the typical elastic waves in the system above the threshold frequency corresponding to the wavelength equivalent to the periodicity of scatterers. (C) 2012 American Institute of Physics. [doi:10.1063/1.3677997]
\end{abstract}

\section{INTRODUCTION}

Recent nanofabrication technology enables us to make a nanometer-scale structure which is separated from the substrate, which is useful to confine electrons in a very small region. $^{1-8}$ The ultimate achievement would produce the single-electron devices that can operate at room temperature. On the other hand, the separation from the substrate makes the nanoscale structure mechanically flexible, and then the structures are deformed with ease and/or vibrate by electric or mechanical forces. A single electron device coupled with mechanical vibrations of the system is referred to as a nanoelectromechanical system (NEMS), which is exploited, e.g., for mass or displacement detection in a quantum limit. ${ }^{9-13}$

Because the deformation of nanoscale structure significantly affects electric characteristics of the device such as capacitances, uncontrolled vibrations will lead to low performance of the device. Then it is necessary to keep the device from the vibrations coming from the environment, and also to transfer redundant vibrations to the circumstances; in another word, one needs to rectify vibrations between the system and the circumstances.

Thermal rectification attracts a lot of attention and the mechanisms are discussed in many works since heat also gives rise to low-performance of the devices. ${ }^{14-30}$ Because the vibrations inducing the structural deformation have a lower frequency than thermal phonons and propagate ballistically in comparison with them, we need an elastic-wave rectifier ${ }^{31-36}$ rather than the thermal rectifier in order to achieve the expected performance of the nano-device, NEMS and so on.

In Refs. 34 and 35, the present authors proposed a rectifier for bulk acoustic waves, and numerically confirmed that the rectifier shows a prominent performance. The rectifier consists of a periodic array of triangular holes drilled in a bulk material, which is the same as Fig. 1(a) but of infinite thickness; $d \rightarrow \infty$. Based on the geometrical acoustics or in the very short wavelength limit [Fig. 1(b)], the incident bulk waves are expected to be specularly scattered by the surfaces of the triangular holes. Supposing that the incident directions

${ }^{a)}$ Electronic mail: yuki@eng.hokudai.ac.jp. are parallel to the $x$ axis, the transmission rate when the incident waves travel toward the vertices of the triangles is larger than that when the waves travel to the bases of them. For acoustic waves with a finite wavelength, it is neither that the geometrical acoustic approximation is available nor that the geometric effects on wave scattering were expected. However, we found numerically that the $z$-polarized transverse acoustic (TA) waves are well rectified by the scatterers. ${ }^{34,35}$ The rectification mechanism is as follows; because of the periodic arrangement of scatterers, the $y$-component of wave vector is allowed to be discrete; $G_{y, n}=n \pi / a(n=0, \pm 1, \pm 2, \ldots)$, so that the dispersion relation of the acoustic waves has a subband structure, i.e., $\omega_{n}=v_{T A} \sqrt{q_{x}^{2}+G_{y, n}^{2}}$, where $v_{T A}$ is the velocity of a transverse bulk wave. The triangular holes cause scattering of the incident wave with $q_{y}=0$, on which there are two constraints; the scattered wave should belong to one of the subbands, and the frequency must be the same as that of the incident wave because of the elastic scattering. When the frequency is lower than $\left.\omega_{ \pm 1}\right|_{q_{x}=0}$, the scattered wave belongs only to the lowest and same subband as that of the incident wave, resulting in forward and backscattering. The transmission rate through the scatterers does not depend on the incident directions, and then there is not a rectification effect in the frequency region. Considering the scattering as an intrasubband transition, an inter-subband transition takes place above the threshold frequency $\left.\omega_{ \pm 1}\right|_{q_{x}=0}$, where the waves are scattered to the directions with $G_{y, \pm 1}$. The distribution of scattered directions for inter-subband transitions depend on the direction of incident waves, i.e., $q_{x}>0$ or $q_{x}<0$ thanks to the asymmetric scatterers, resulting in rectification. Thus, the rectification is due to the collaboration of interference effects among scattered waves and geometric effects of asymmetric scatterers. This is distinct from the rectification mechanisms utilizing nonlinear effects studied in the previous works. $^{31-33}$

In contrast to the $z$-polarized TA waves, the rectification effects are not obvious for the longitudinal (LA) and TA bulk waves polarized normally to the hole axis, since the mode conversion between LA and TA waves at the hole surfaces weakens the rectification effects. ${ }^{36}$ 
In the present work, considering that a suspended structure is often used in NEMS, we apply the model to a freestanding thin plate [Fig. 1(a)] and investigate the propagation of elastic waves. Lamb waves are typical acoustic waves in a thin plate, which are composed of LA and TA waves so that they satisfy stress-free boundary condition on the surface. The particle motion is limited in the sagittal plane. But the LA wave component will be converted to TA one for scattering, which is likely to weaken the rectification effects of the Lamb waves. In the present work, we elucidate the characteristic transmission behaviors of Lamb waves as well as shear horizontal (SH) waves due to the periodic asymmetric scatterers. The paper is organized as follows; we introduce a model of rectifier for Lamb waves, and provide the methodology based

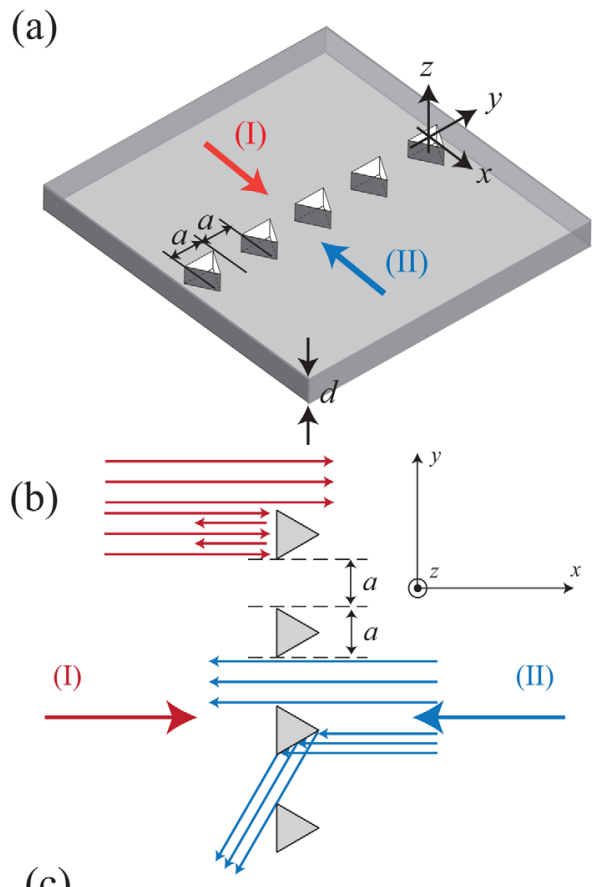

(c)

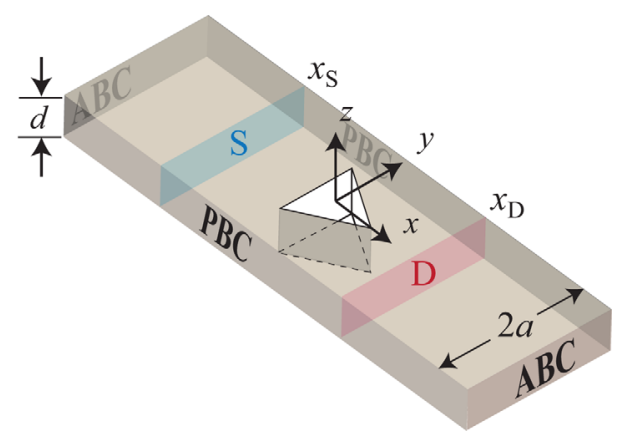

FIG. 1. (Color online) (a) Model of the acoustic wave rectifier. This is made of an elastically isotropic plate of $d$ in thickness and triangular holes equally spaced by the base length $a$ in the $y$ direction. (b) Acoustic ray propagation based on the geometrical acoustics. The transmission through the line of scatterers depends on the incident directions. The thick arrows indicate the directions (I) and (II) of incident waves, and the thin arrows show reflection and transmission of waves. (c) Unit cell used in the simulation. The unit cell has a triangular scatter at the origin, and is subject to the periodic boundary condition (PBC) and the absorbing boundary condition ( $\mathrm{ABC}$ ). The PBCs are imposed on both the surfaces normal to the $y$ direction along the array and the $\mathrm{ABCs}$ are used on both the end surfaces normal to the $x$ direction. $S$ and $D$ indicate the cross section for excitation and detection of acoustic waves when the incident waves impinge first on the base of the scatterer [case (I)]. The positions are switched for case (II). on the finite-difference time-domain (FDTD) scheme in Sec. II. A brief survey of Lamb waves in a homogeneous thin plate is made in this section. In Sec. III, the numerical results for antisymmetric, symmetric Lamb, and shear horizontal waves in the proposed rectifier are given. Finally, a summary is made in Sec. IV.

\section{MODEL AND FORMALIZATION}

Figure 1(a) shows the rectifier that we investigate in the present work, which is an elastically isotropic thin plate of $d$ in thickness having an array of triangular holes as scatterers. The geometry of the holes is an equilateral triangle of base length $a$. As we are interested in the difference in transmission behaviors for wave propagation in the $x$ direction, we refer, hereafter, the case that incident waves impinge first on the bases to as 'case (I)' and that on the summits to as 'case (II),' respectively.

The equation of motion for the displacement vector $\mathbf{u}(\mathbf{r}, t)$ and Hooke's law are given by

$$
\begin{gathered}
\rho(\mathbf{x}) \ddot{u}_{i}(\mathbf{r}, t)=\partial_{j} \sigma_{i j}(\mathbf{r}, t), \\
\sigma_{i j}(\mathbf{r}, t)=c_{i j m n}(\mathbf{x}) \partial_{n} u_{m}(\mathbf{r}, t),
\end{gathered}
$$

where $\mathbf{r}=(\mathbf{x}, z)=(x, y, z)$ and the summation convention over repeated indices is assumed in Eqs. (1) and (2). $\rho(\mathbf{x})$ and $c_{i j m n}$ (x) are the position-dependent mass density and elastic stiffness tensor of the system, and $\sigma_{i j}(\mathbf{r}, t)$ is the stress tensor. Acoustic waves are subject to the stress-free boundary conditions at the surfaces $z= \pm d / 2$, or

$$
\left.\sigma_{i z}\right|_{z= \pm d / 2}=\left.c_{i z m n} \partial_{n} u_{m}\right|_{z= \pm d / 2}=0
$$

and are classified into the SH and Lamb modes. Based on the parity for inversion about the $z=0$ plane, the Lamb mode is also classified into symmetric Lamb (SL) and anti-symmetric Lamb (ASL) modes.

The dispersion relations for the Lamb waves are derived from a set of equations known as the Rayleigh-Lamb equations, which are given by

$$
\frac{\tan \left(k_{z} d / 2\right)}{\tan \left(q_{z} d / 2\right)}=-\left[\frac{4 q_{x}^{2} q_{z} k_{z}}{\left(k_{z}^{2}-q_{x}^{2}\right)^{2}}\right]^{ \pm 1}
$$

for the SL mode (plus sign) and the ASL mode (minus sign), respectively. Here $\mathbf{q}=\left(q_{x}, 0, q_{z}\right)$ and $\mathbf{k}=\left(q_{x}, 0, k_{z}\right)$ are the wave vectors of LA and TA waves in a saggital $(x-z)$ plane, respectively, and $q_{x}, q_{z}$, and $k_{z}$ satisfy the following dispersion relations:

$$
\omega^{2}=v_{l}^{2}\left(q_{x}^{2}+q_{z}^{2}\right)=v_{t}^{2}\left(q_{x}^{2}+k_{z}^{2}\right),
$$

where $v_{l}$ and $v_{t}$ are the velocities of bulk longitudinal and transverse waves, respectively. The dispersion relation for the $\mathrm{SH}$ waves yields

$$
\omega=v_{t} \sqrt{q_{x}^{2}+\left(\frac{2 \pi n}{d}\right)^{2}}(n=0, \pm 1, \pm 2, \cdots) .
$$


Figure 2 shows the dispersion relations for the three modes in a homogeneous thin plate composed of tungsten. Tungsten is elastically isotropic, where the mass density $\rho_{\mathrm{W}}$ and the elastic stiffness tensors $C_{11}^{\mathrm{W}}, C_{44}^{\mathrm{W}}$ are $19.317 \mathrm{~g} / \mathrm{cm}^{3}$ and $5.326 \times 10^{12} \mathrm{dyn} / \mathrm{cm}^{2}, 1.631 \times 10^{12} \mathrm{dyn} / \mathrm{cm}^{2}$, respectively. ${ }^{37}$ The sound velocities of bulk LA and TA waves are $v_{l}=5.25 \times 10^{3} \mathrm{~m} / \mathrm{s}$ and $v_{t}=2.906 \times 10^{3} \mathrm{~m} / \mathrm{s}$. As is well known, the dispersion curves of the lowest SL and SH modes are linear with respect to the wave vector $q_{x}$ in the lowfrequency limit, while the dispersion curve of the lowest ASL mode increases quadratically. The dispersion curves at higher frequencies are the subband structures originating from confinement of the modes in the $z$ direction. ${ }^{38}$

In order to investigate the transmission properties of the acoustic waves through the periodic array of asymmetric scatterers, we employ a FDTD method. In order to save the memory for numerical calculations, we introduce a unit cell which contains a scatterer at the origin as shown in Fig. 1(c), and deal with that as a building block. We request that each unit cell is subject to the periodic boundary condition (PBC) on both the surfaces normal to the $y$ direction and to the absorbing boundary condition $(\mathrm{ABC})$ on both the end surfaces normal to the $x$ direction.

Solving Eqs. (1) and (2) numerically, we obtain the displacement vector $\mathbf{u}(\mathbf{r}, t)$ and stress tensor $\sigma_{i j}(\mathbf{r}, t)$ at each grid point in the unit cell. To calculate the transmission rate through the scatterers, we introduce the acoustic Poynting vector defined by $J_{i}(\mathbf{r}, t)=-\dot{u}_{j}(\mathbf{r}, t) \sigma_{j i}(\mathbf{r}, t)$ from the continuity of elastic energy flow. Using the Fourier components of the displacement $\hat{\mathbf{u}}(\mathbf{r}, \omega)$ and the stress tensor $\hat{\sigma}_{i j}(\mathbf{r}, \omega)$, the energy flow spectrum $J_{x}(x, \omega)$ in the $x$ direction at the position $x$ is expressed by

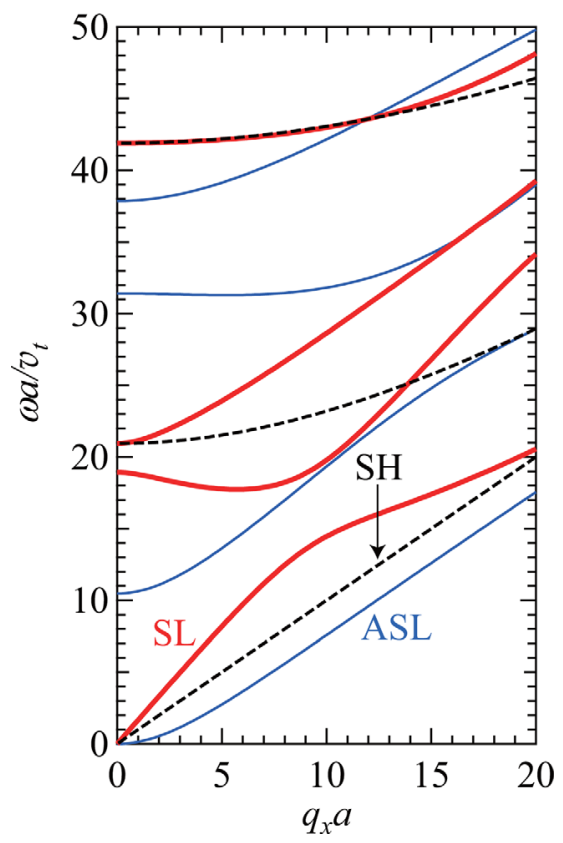

FIG. 2. (Color online) Dispersion relations for acoustic waves in a thin plate. The symmetric Lamb (SL), anti-symmetric Lamb (ASL), and shearhorizontal (SH) modes are denoted by thick, thin, and dashed lines, respectively.

$$
J_{x}(x, \omega)=-4 \pi \int_{D} \operatorname{Im}\left[\omega \hat{u}_{j}(\mathbf{r}, \omega) \hat{\sigma}_{j x}^{*}(\mathbf{r}, \omega)\right] d y d z
$$

where $D$ indicates the cross-section area of the $y-z$ plane in Fig. 1(c). It should be noted that the energy flux $J_{x}(x, \omega)$ in the frequency domain defined here is not the mere Fourier transform of the Poynting vector $J_{x}(x, t)$ in the time domain (see Appendix). Here we estimate the transmission rate $T(\omega)$ by the ratio of $J_{x}\left(x_{D}, \omega\right)$ to that in the absence of scatterers $J_{x}^{0}\left(x_{D}, \omega\right)$,

$$
T(\omega)=\frac{J_{x}\left(x_{D}, \omega\right)}{J_{x}^{0}\left(x_{D}, \omega\right)},
$$

where $x_{D}$ is the detecting position which is behind the scatterers, i.e., $x_{D}>0$ for case (I) and $x_{D}<0$ for case (II). The origin of the $\mathrm{x}$ axis is set to the centroid of a triangular hole. For both the cases $\left|x_{D}\right|=3 a$ in our simulation. In order to quantify the rectification effects, we define the efficiency $\eta(\omega)$ by

$$
\eta(\omega)=\left|\frac{T_{(\mathrm{I})}(\omega)-T_{(\mathrm{II})}(\omega)}{T_{(\mathrm{I})}(\omega)+T_{(\mathrm{II})}(\omega)}\right|,
$$

where $T_{(\mathrm{I})}$ and $T_{\text {(II) }}$ are the transmission rates for the cases (I) and (II), respectively.

\section{NUMERICAL RESULTS}

We illustrate the rectification effects of Lamb waves, applying our model to a tungsten plate whose thickness is set to be $d=0.3 a$. The stiffness tensor in the holes should be put $C_{11}^{\mathrm{vac}}=C_{44}^{\mathrm{vac}}=0$. The mass density for vacuum $\rho_{\mathrm{vac}}$ should be zero, too, however, we actually set a finite mass density for vacuum $\rho_{\mathrm{vac}}=\rho_{\mathrm{W}} / 100$ because of technical reasons for the FDTD method. ${ }^{39}$

We first investigate the transmission behaviors of ASL waves. In order to excite an incident wave packet, we shake the cross section at $S$ in Fig. 1(c) in the $z$ direction at a frequency of purpose, and thereby make a $z$-polarized wave packet by setting an appropriate excitation time interval. ${ }^{40}$ The in-phase motion of the cross section is useful to generate a wave that propagates in the $x$ direction, which belongs to the lowest subband of the ASL waves in Fig. 2 since the lowest subband of the ASL waves does not have a node in the $z$ direction.

Figure 3(a) shows the transmission rates versus frequency for the ASL waves. ${ }^{41}$ It is found that there is no difference in the transmission rates between the two opposite incident directions in the frequency region lower than $\omega a / v_{t}$ $\approx 1.35$. In contrast, the obvious rectification effects for ASL waves appear in the entire frequency region above the frequency. In addition, we also find the dips in the transmission rates that appear periodically with increasing frequency.

In order to elucidate the origin of the threshold frequency and dips in transmission rates, we compare the transmission rates with the dispersion relations for ASL waves [Fig. 3(b)] by means of the empty-lattice approximation (ELA), considering the periodic structure in the $y$ direction. Based on the 
ELA, we replace $q_{x}$ by $\sqrt{q_{x}^{2}+(n \pi / a)^{2}}(n=0, \pm 1, \pm 2, \cdots)$ in Eq. (4), where $n \pi / a$ is the reciprocal vector in the $y$ direction. Within the approximation the dispersion relation has a subband structure due to the periodicity $2 a$ in the $y$ direction in addition to the subbands due to the confinement in the $z$ direction. Figure 3(b) shows the subbands originating from the dispersion relations of the lowest one of ASL waves in Fig. 2 as well as the second one.

We find that there are clear correspondences between the frequencies at which the dips in the transmission rates appear and those of the subband bottoms of the dispersion relations. The wave vector of the ASL waves in the $y$ direction is finite except for the lowest subband or the incident waves. The dips in the transmission rates indicate the intersubband transition from the incident wave to another subband having a finite $y$-component of wave vector. Since the subband bottoms are flat with respect to $q_{x}$, the density of the final states for the scattering diverges. Then the intersubband transitions take place extremely at the frequencies, resulting in the dips in the transmission rates. The difference in the transmission rates between the opposite incident directions is due to the geometric effects of scattering caused by the asymmetric scatterers, giving rise to the rectification of the ASL waves.

The rectification does not appear below the threshold frequency as mentioned above. The lowest dip in the transmission rates appears at the bottom of the second subband. Below the frequency, only the lowest subband is available for the scattering. Then the scattering causes intra-subband transitions, i.e., backscattering of the incident waves. Because the backscattering equally occurs for both the incident directions, there is no difference in the transmission rates below the second subband. Then the frequency of the second subband gives the threshold frequency, $\omega a / v_{t}=(d / a) \pi^{2} \sqrt{\left(\kappa^{2}-1\right) / 3 \kappa^{2}}$

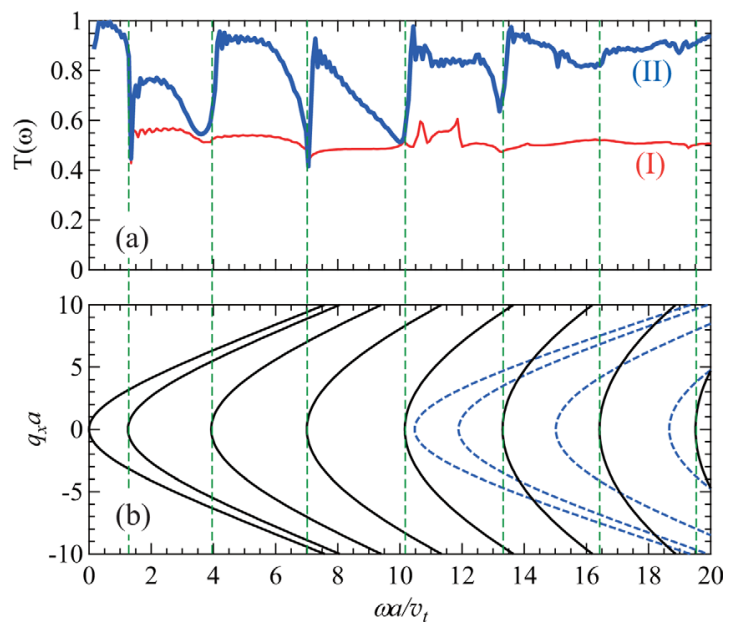

FIG. 3. (Color online) (a) Transmission rates vs frequency for the ASL waves through a single-array of triangular holes. The directions of the incident waves are designated by (I) thin (red) and (II) thick (blue) solid lines, respectively. (b) Dispersion relations for the ASL waves within the ELA. The dispersion curves denoted by solid lines stem from the lowest subband of the ASL waves in Fig. 2, and those by dashed lines stem from the second subband of the ASL waves. The vertical dashed lines indicate the frequencies of the bottoms of the subband belonging to the ASL waves. $\approx 1.42\left(\kappa=v_{l} / v_{t}\right)$, which agrees with the numerical simulations.

As mentioned above, Fig. 3(b) contains the subbands originating from the second dispersion relation of the ASL waves in Fig. 2. The waves belonging to the subbands have two nodes in the $z$ direction, then the inter-subband transitions from the lowest subband having homogeneous displacement in the $z$ direction are expected to be small. Although Fig. 3(a) shows two peaks at $\omega a / v_{t}=10.65$ and 11.85 in the transmission rates for case (I) and a dip at 15.08 for case (II), the changes in the transmission rates are not noticeable, as expected.

Figure 4(a) shows the transmission rates versus frequency for the SL waves. In order to excite a wave packet of SL waves, the cross section $S$ is driven, at a frequency of interest, in the $x$ direction within a certain time interval. ${ }^{40}$ It is found that in the frequency region lower than $\omega a / v_{t} \approx 3.14$ the transmission rates of the cases (I) and (II) coincide. Above the frequency, the transmission rates show the obvious rectification effects, and the frequency dependence is found to be complicated in comparison with the ASL waves. Another noticeable point is that the threshold frequency is larger than that of ASL waves.

The rectification mechanism is essentially the same as the ASL waves, however, the contribution from the $\mathrm{SH}$ waves is significant for the rectification. In order to see the contribution, we plot the dispersion relations for both the SL and SH waves within the ELA in Fig. 4(b). The lowest solid line is the dispersion relation of the incident wave, and the dashed lines are those of the SH waves. The lowest dip in the transmission rates appears at the bottom frequency of the $\mathrm{SH}$ waves, which becomes the threshold frequency. In the frequency region larger than the threshold frequency, the dips at $\omega a / v_{t}=6.38,9.53$, and 12.20 are due to mode conversions from the SL waves to the SH ones. On the other hand, no noticeable dips appear at the frequencies of the subband bottoms of SL waves. Thus, the contribution of the inter-subband

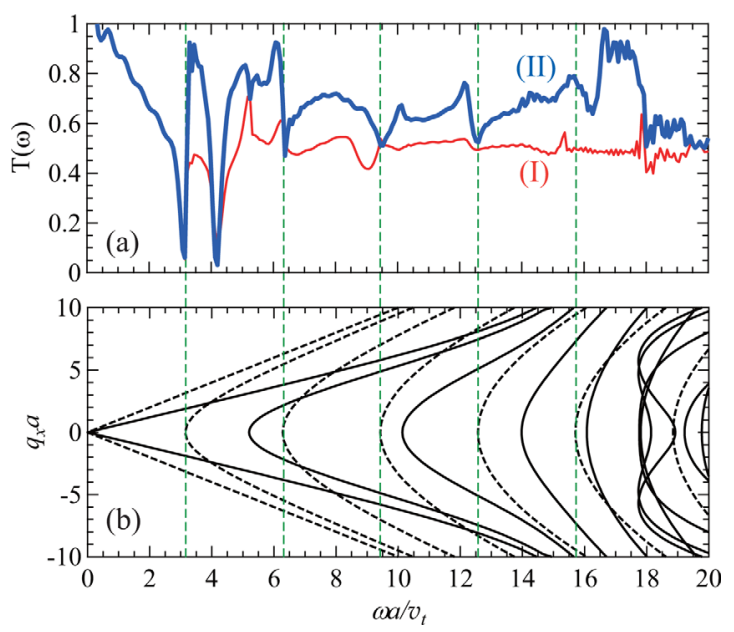

FIG. 4. (Color online) (a) Transmission rate vs frequency for the SL waves through a single-array of triangular holes. The directions of the incident waves are designated by (I) thin (red) and (II) thick (blue) solid lines, respectively. (b) Dispersion relations for the SL (solid lines) and SH waves (dashed lines) within the ELA. The vertical dashed lines indicate the frequencies at the subband bottoms belonging to the $\mathrm{SH}$ mode. 
transitions among the SL waves to the rectification is extremely limited in comparison with the mode conversion from the SL waves to the SH ones.

Although the dips in the transmission rates are connected with the band structures, the dip at $\omega a / v_{t}=4.20$ cannot be related to a mode conversion since there is not a corresponding subband bottom. At the frequency, the dispersion curve of the incident wave and the second dispersion curve of SH waves intersect. These waves are converted to each other via scattering, and therefore we expect modecoupling of the SL and SH waves at the frequency. As a consequence, a frequency bandgap will open at the frequency, omitting the transmission of the incident wave. Although such a gap is known to create a bandgap in the superlattice structure, we need further study to confirm the speculation.

We have found that the mode conversion plays a significant role in the rectification mechanism rather than the intersubband transitions among the SL modes. To make the effects of mode conversion clear, the transient energy distribution of the wave scattered by a single triangular hole is illustrated in Fig. 5. The energy distribution is defined by $E(t)=\Sigma_{i}\left|u_{i}(t)\right|{ }^{2}$ When the incident plane wave impinges on the triangular summit, Fig. 5(a) shows that most part of scattered waves travels in the $y$ direction as the $\mathrm{SH}$ waves rather than the SL waves. The inset shows the propagation direction of each wave. On the other hand, when the incident plane wave impinges on the triangular base, Fig. 5(b) shows that most part of scattered waves is reflected by the base and returns as SL waves without mode conversion. Thus, the mode conversion from the SL waves to SH ones occurs remarkably in comparison with the scattering to other SL modes. Because of that, the rectification effects for the SL waves depend predominantly on the mode conversion as seen in Fig. 4.

From the roles of the mode conversion between the SL and $\mathrm{SH}$ waves in the rectification mechanism, we also expect that the transmission rates of $\mathrm{SH}$ waves depend on the mode conversion. On the other hand, the mode conversion from the $\mathrm{SH}$ waves to the SL waves is not expected when the SH waves impinge on the slope of the triangle since the wave number parallel to the slope is not conserved for the SL waves. The incident SH waves will be reflected specularly from the slope.

Figure 6 shows the transmission rates versus frequency when the incident wave is a $\mathrm{SH}$ wave. The transmission rates show the threshold frequency and rectification effects above it. It corresponds to the frequency of the second subband bottom of the SH waves. However, not all the dips in the transmission rates are related to the SH subband bottoms, and the dip at $\omega a / v_{t}=5.2$ coincides with the bottom of the SL waves, showing possible mode conversion from the SH waves to SL waves in the present system. One of the remarkable behaviors is the inversion of the transmission rates between cases (I) and (II) in the frequency region $8<\omega a / v_{t}<9$, contrary to our expectations. The rectification mechanism is based on the strong wave scattering in this system, and then multiple scattering processes of waves might cause such unexpected transmission properties.

Finally, we estimate the performance of the rectifier for the Lamb waves. Figure 7 plots the efficiency $\eta(\omega)$ defined
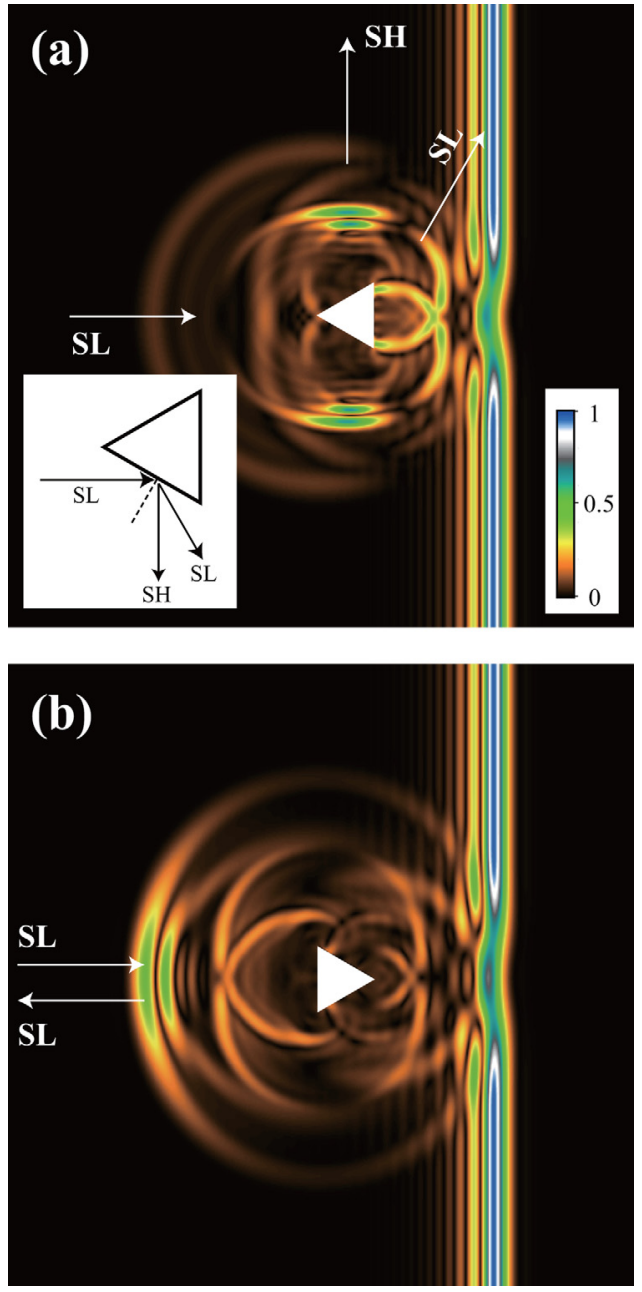

FIG. 5. (Color online) Transient energy distribution after scattering of a plane wave packet by a single triangular hole. (a) and (b) indicate the cases of the wave packets incident on the summit and the base of a triangular hole, respectively. The energy distribution is defined by $E(\mathbf{r}, t)=\Sigma_{i}\left|u_{i}(\mathbf{r}, t)\right|{ }^{2}$ The inset of (a) indicates the propagation directions of the incident, and scattered SL and SH waves.

by Eq. (9) for the (a) SL, (b) ASL, and (c) SH waves. According to the geometric acoustics, $\eta_{g a}=1 / 3$, and $\eta$ for all the modes is lower than $\eta_{\mathrm{ga}}$ in the entire frequency region, except for the peaks at $\omega a / v_{t}=4.20$ for the SL waves, and at $\omega a / v_{t}=3.14,6.08$, and 9.15 for the SH waves. Among the three modes, the ASL waves show modest frequency dependence of $\eta$ close to $\eta_{\mathrm{ga}}$, and the threshold frequency for the ASL waves is lower than those of the SL and SH waves. Considering these characteristics, the present rectifier shows better performance for the ASL mode.

\section{SUMMARY}

We proposed the rectifier for acoustic waves propagating in a thin plate, which is made of an elastic material containing an array of triangular holes. The typical modes peculiar to the thin plate are the $\mathrm{SH}$ and symmetric and anti-symmetric Lamb modes, and we investigated the propagation properties of the waves by numerical simulations with a FDTD method. The transmission rates depend on the incident directions, showing obvious differences in magnitude as well as the 


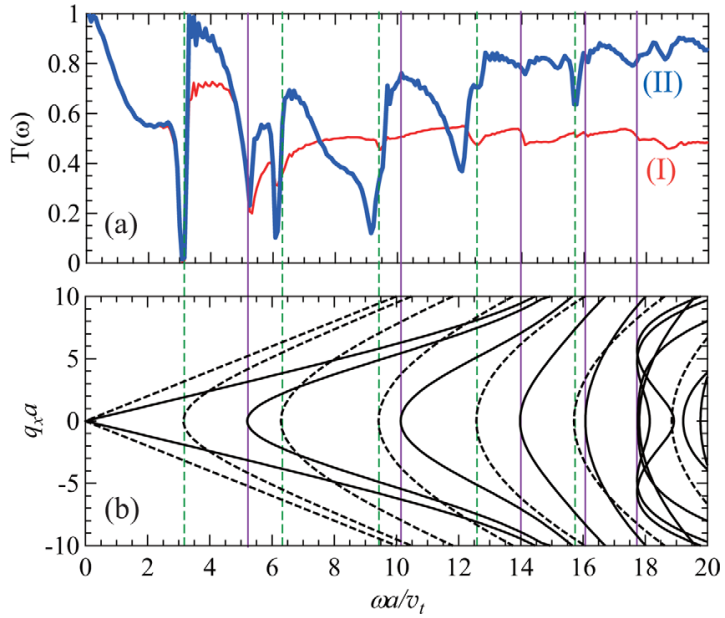

FIG. 6. (Color online) Transmission rate vs frequency for the SH waves through a single-array of triangular holes. The directions of the incident waves are designated by (I) thin (red) and (II) thick (blue) solid lines, respectively. (b) Dispersion relations for the SL (solid lines) and SH waves (dashed lines) within the ELA. The vertical dashed and solid lines indicate the frequencies at the subband bottoms belonging to the SH and SL mode, respectively.

frequency dependences. The differences are brought about by the geometric effects on wave scattering by the asymmetric scatterers, showing that the present system works as a rectifier for the acoustic waves. In addition, the interplay between the mode conversion and the interference effects among the scattered waves complicates it in specified frequency regions. The transmission rates and the efficiency show distinct behaviors for each mode when the incident waves impinge first on the summits of the triangular holes. The rectifier has a low threshold frequency for the anti-symmetric Lamb mode and modest frequency dependence both for the transmission rates and for
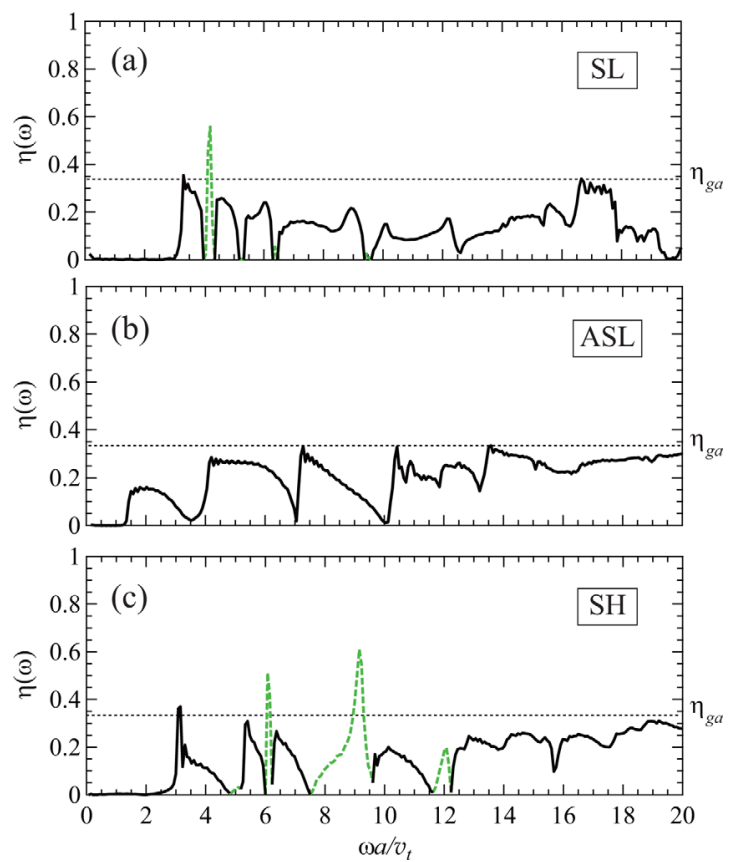

FIG. 7. (Color online) Efficiency $\eta(\omega)$ vs frequency for the SL, ASL, and SH waves. $\eta_{g a}$ is the efficiency within the geometric acoustics approximation. The dashed lines represent the regions where the magnitudes of transmission rates of case (I) and case (II) are inverted contrary to our expectation. the efficiency in comparison with other modes. Considering that flexural vibrations are most easily excited for a thin plate, the system can be used to control elastic vibrations in the nanoscale structures. In contrast, the transmission rates for the SH and symmetric Lamb modes change quickly, depending on frequency. In particular, the transmission rates for the $\mathrm{SH}$ waves decrease rapidly below 0.5 in the frequency region $7<\omega a / v_{t}<9$. The valley of the transmission rates can be utilized as a band-stop filter for acoustic waves. Thus the findings of the present work will yield a clue of the implementation of the control of acoustic waves and the removal of redundant vibrations in a NEMS composed of suspended wires or quantum dots. Furthermore, they can be applied not only to acoustic waves in solids or liquids but also in optical waves, leading to new devices in wave engineering.

\section{ACKNOWLEDGMENTS}

This work was supported in part by a Grant-in-Aid for Scientific Research from the Ministry of Education, Science and Culture of Japan (Grant No. 23560052). The numerical calculations were performed in the supercomputing facilities (HITACHI SR11000) in Hokkaido University.

\section{APPENDIX: DEFINITION OF ENERGY FLUX IN FREQUENCY DOMAIN}

The element of the Poyting vector flowing in the $i$ direction is given by

$$
J_{i}(\mathbf{r}, t)=-\dot{u}_{j}(\mathbf{r}, t) \sigma_{j i}(\mathbf{r}, t)
$$

Therefore, the total energy $E_{i}$ passing through the point $\mathbf{r}$ in the $i$ direction is expressed by

$$
\begin{aligned}
E_{i} & =\int_{-\infty}^{\infty} J_{i}(\mathbf{r}, t) d t \\
& =\int_{-\infty}^{\infty}\left[-\dot{u}_{j}(\mathbf{r}, t) \sigma_{j i}(\mathbf{r}, t)\right] d t .
\end{aligned}
$$

Substituting $u_{j}(\mathbf{r}, t)$ and $\sigma_{j i}(\mathbf{r}, t)$ in terms of the Fourier transform

$$
\begin{gathered}
u_{j}(\mathbf{r}, t)=\int_{-\infty}^{\infty} \hat{u}_{j}(\mathbf{r}, \omega) e^{-i \omega t} d \omega, \\
\sigma_{j i}(\mathbf{r}, t)=\int_{-\infty}^{\infty} \hat{\sigma}_{j i}(\mathbf{r}, \omega) e^{-i \omega t} d \omega,
\end{gathered}
$$

into Eq. (A2), we obtain

$$
\begin{aligned}
E_{i} & =2 \pi i \int_{-\infty}^{\infty} \omega \hat{u}_{j}(\mathbf{r}, \omega) \hat{\sigma}_{j i}(\mathbf{r},-\omega) d \omega \\
& =-4 \pi \int_{0}^{\infty} \operatorname{Im}\left\{\omega \hat{u}_{j}(\mathbf{r}, \omega) \hat{\sigma}_{j i}^{*}(\mathbf{r}, \omega)\right\} d \omega,
\end{aligned}
$$

where we use the relation that $\sigma_{i j}^{*}(\mathbf{r}, \omega)=\sigma_{i j}(\mathbf{r},-\omega)$ and $u_{i}^{*}(\mathbf{r}, \omega)=u_{i}(\mathbf{r},-\omega)$ since the displacements and stresses are real. From the result, the $i$ th element of the energy flow $\hat{J}_{i}(\mathbf{r}, \omega)$ in the frequency domain yields 


$$
J_{i}(\mathbf{r}, \omega)=-4 \pi \operatorname{Im}\left\{\omega \hat{u}_{j}(\mathbf{r}, \omega) \hat{\sigma}_{j i}^{*}(\mathbf{r}, \omega)\right\}
$$

Therefore, the energy flux passing through the cross section at $x$ is expressed by

$$
J_{x}(x, \omega)=-4 \pi \int_{D} \operatorname{Im}\left\{\omega \hat{u}_{j}(\mathbf{r}, \omega) \hat{\sigma}_{j x}^{*}(\mathbf{r}, \omega)\right\} d y d z .
$$

${ }^{1}$ C. S. Yung, D. R. Schmidt, and A. N. Cleland, Appl. Phys. Lett. 81, 31 (2002).

${ }^{2}$ W. Fon, K. C. Schwab, J. M. Worlock, and M. L. Roukes, Phys. Rev. B 66, 045302 (2002).

${ }^{3}$ L. Sainiemi, K. Grigoras, and S. Franssila, Nanotechnology 20, 075306 (2009).

${ }^{4}$ C. C. Huang and K. L. Ekinci, Appl. Phys. Lett. 88, 093110 (2006).

${ }^{5}$ C. Meyer, O. Sqalli, H. Lorenz, and K. Karrai, 4th IEEE Conference on Nanotechnology, 435 (2004).

${ }^{6}$ E. M. Weig, R. H. Blick, T. Brandes, J. Kirschbaum, W. Wegscheider, M. Bichler, and J. P. Kotthaus, Phys. Rev. Lett. 92, 046804 (2004).

${ }^{7}$ A. G. Pogosov, M. V. Budantsev, R. A. Lavrov, A. E. Plotnikov, A. K. Bakarov, A. I. Toropov, and J. C. Portal, JETP Lett. 83, 152 (2006).

${ }^{8}$ A. G. Pogosov, M. V. Budantsev, A. A. Shevyrin, A. E. Plotnikov, A. K. Bakarov, and A. I. Toropov, JETP Lett. 90, 626 (2009).

${ }^{9}$ N. V. Lavrik and P. G. Datskos, Appl. Phys. Lett. 82, 2697 (2003).

${ }^{10}$ K. L. Ekinci, X. M. H. Huang, and M. L. Roukes, Appl. Phys. Lett. 84, 4469 (2004).

${ }^{11}$ A. Gupta, D. Akin, and R. Bashir, Appl. Phys. Lett. 84, 1976 (2004).

${ }^{12}$ K. L. Ekinci, Y. T. Yang, X. M. H. Huang, and M. L. Roukes, Appl. Phys. Lett. 81, 2253 (2002).

${ }^{13}$ F. W. Beil, L. Pescini, E. Höhberger, A. Kraus, A. Erbe, and R. H. Blick, Nanotechnology 14, 799 (2003).

${ }^{14}$ M. Terraneo, M. Peyrard, and G. Casati, Phys. Rev. Lett. 88, 094302 (2002).

${ }^{15}$ B. Li, L. Wang, and G. Casati, Phys. Rev. Lett. 93, 184301 (2004).

${ }^{16}$ L.-A. Wu and D. Segal, Phys. Rev. Lett. 102, 095503 (2009).

${ }^{17}$ W. C. Lo, L. Wang, and B. Li, J. Phys. Soc. Jpn. 77, 054402 (2008).

${ }^{18}$ M. Alaghemandi, F. Leroy, F. Müller-Plathe, and M. C. Böhm, Phys. Rev. B 81, 125410 (2010).

${ }^{19}$ M. Hu, J. V. Goicochea, B. Michel, and D. Poulikakos, Appl. Phys. Lett. 95, 151903 (2009).

${ }^{20}$ T. Ruokola, T. Ojanen, and A.-P. Jauho, Phys. Rev. B 79, 144306 (2009).
${ }^{21}$ C. Xue-Ou, D. Bing, and L. Xiao-Lin, Chin. Phys. Lett. 25, 3032 (2008).

${ }^{22}$ B. Li and J. Wang, Phys. Rev. Lett. 91, 044301 (2003).

${ }^{23}$ M. Peyrard, Europhys. Lett. 76, 49 (2006).

${ }^{24}$ W. Kobayashi, Y. Teraoka, and I. Terasaki, Appl. Phys. Lett. 95, 171905 (2009).

${ }^{25}$ C. W. Chang, D. Okawa, A. Majumdar, and A. Zettl, Science 314, 1121 (2006).

${ }^{26}$ A. M. Song, A. Lorke, A. Kriele, J. P. Kotthaus, W. Wegscheider, and M. Bichler, Phys. Rev. Lett. 80, 3831 (1998).

${ }^{27}$ R. Fleischmann and T. Geisel, Phys. Rev. Lett. 89, 016804 (2002).

${ }^{28}$ H. Linke, W. Sheng, A. Löfgren, H.-G. Xu, P. Omling, and P. E. Lindelof, Europhys. Lett. 44, 341 (1998).

${ }^{29}$ B. Li, J. Lan, and L. Wang, Phys. Rev. Lett. 95, 104302 (2005).

${ }^{30}$ B. Li, L. Wang, and G. Casati, Appl. Phys. Lett. 88, 143501 (2006).

${ }^{31}$ B. Liang, B. Yuan, and J.-C. Cheng, Phys. Rev. Lett. 103, 104301 (2009).

${ }^{32}$ B. Liang, X. S. Guo, J. Tu, D. Zhang, and J. C. Cheng, Nature Materials 9 , 989 (2010).

${ }^{33}$ S. Lepri and G. Casati, Phys. Rev. Lett. 106, 164101 (2011).

${ }^{34}$ R. Krishnan, S. Shirota, Y. Tanaka, and N. Nishiguchi, Solid State Communications 144, 194 (2007).

${ }^{35}$ S. Shirota, R. Krishnan, Y. Tanaka, and N. Nishiguchi, Japanese Journal of Applied Physics 46, L1025 (2007).

${ }^{36}$ Y. Tanaka, T. Murai, and N. Nishiguchi, in Acoustic Waves, edited by D. W. Dissanayake (SCIYO book, 2010), Chap 3.

${ }^{37}$ C. Kittel, Introduction to Solid State Physics, 8th ed. (Wiley, New York, 2004).

${ }^{38}$ K. F. Graff, Wave Motion in Elastic Solids (Dover Publications, New York, 1975).

${ }^{39}$ Our results did not depend on any $\rho_{\text {vac }}$ in this order in the frequency domain of interest. Therefore, we are convinced that vacuum can be expressed by $\rho_{\text {vac }}=\rho_{\mathrm{w}} / 100$

${ }^{40}$ For the purpose of making a wave packet of symmetric or dilatational Lamb waves, we vibrate the $S$-plane in the $x$-direction. The driving frequency is fixed at the central frequency, and the driving amplitude changes gradually with time and has a peak at $t=0$. That is, $f=A \exp \left(-\alpha t^{2}\right) \sin (\omega t)$. The envelop of the driving amplitude provides spectral width $1 / \sqrt{\alpha}$, which is adjusted in order to obtain data for the entire frequency region of interest. The excited wave packet is regarded as superposition of symmetric Lamb waves with different frequencies. As for the excitation of antisymmetric Lamb waves, we drive the $S$-plane in the $z$ direction in the same manner as the symmetric Lamb wave excitation.

${ }^{41}$ In our simulation, the grid size used is $\Delta x=\Delta y=\Delta z=a / 50$, and the time interval is $\Delta t=1.0 \times 10^{-3} a / v_{t}$ 